\title{
Protective Effect of Ligustrazine on Lumbar Intervertebral Disc Degeneration of Rats Induced by Prolonged Upright Posture
}

\author{
Qian-Qian Liang, ${ }^{1,2}$ Dao-Fang Ding, ${ }^{1,2}$ Zhi-Jie Xi, ${ }^{1,2}$ Yan Chen, ${ }^{1,2}$ Chen-Guang Li, ${ }^{1,2}$ \\ Shu-Fen Liu, ${ }^{1,2}$ Sheng Lu, ${ }^{1,2}$ Yong-Jian Zhao, ${ }^{1,2}$ Qi Shi, ${ }^{1,2}$ and Yong-Jun Wang ${ }^{1,2}$ \\ ${ }^{1}$ Institute of Spine, Shanghai University of Traditional Chinese Medicine, 725 Wan-Ping South Road, Shanghai 200032, China \\ ${ }^{2}$ Department of Orthopaedics \& Traumatology, Longhua Hospital, Shanghai University of Traditional Chinese Medicine, \\ Shanghai, China \\ Correspondence should be addressed to Yong-Jun Wang; yjwang8888@126.com
}

Received 19 November 2013; Revised 3 April 2014; Accepted 9 April 2014; Published 29 April 2014

Academic Editor: I-Min Liu

Copyright (C) 2014 Qian-Qian Liang et al. This is an open access article distributed under the Creative Commons Attribution License, which permits unrestricted use, distribution, and reproduction in any medium, provided the original work is properly cited.

Most chronic low back pain is the result of degeneration of the lumbar intervertebral disc. Ligustrazine, an alkaloid from Chuanxiong, reportedly is able to relieve pain, suppress inflammation, and treat osteoarthritis and it has the protective effect on cartilage and chondrocytes. Therefore, we asked whether ligustrazine could reduce intervertebral disc degeneration. To determine the effect of ligustrazine on disc degeneration, we applied a rat model. The intervertebral disc degeneration of the rats was induced by prolonged upright posture. We found that pretreatment with ligustrazine for 1 month recovered the structural distortion of the degenerative disc; inhibited the expression of type X collagen, matrix metalloproteinase (MMP)-13, and MMP3; upregulated type II collagen; and decreased IL-1 $\beta$, cyclooxygenase (COX)-2, and inducible nitric oxide synthase (iNOS) expression. In conclusion, ligustrazine is a promising agent for treating lumbar intervertebral disc degeneration disease.

\section{Introduction}

Chronic low back pain, resulting from intervertebral disc degeneration $[1,2]$, is one of the most common and costly musculoskeletal pain syndromes of modern society $[3,4]$. Degeneration of intervertebral discs (IVD) plays a central role in the pathogenesis of discogenic pain, disc herniation, and spinal instability and stenosis $[5,6]$. The IVD health is influenced by the state of the adjacent vertebral endplate. Nutrient diffusion through the endplate to the IVD is critical for the maintenance of normal IVD function [7, 8]. Pathologic changes in endplate cartilage are closely related to IVD degeneration $[9,10]$. Prevention and reduction of lesions in vertebral endplate are therefore a high research priority.

Chuanxiong is a dry root of the Chuanxiong plant. In ancient China this plant was usually used to treat low back pain. Ligustrazine is an alkaloid from Chuanxiong, and its chemical structure is tetramethylpyrazine. Currently it can be synthesized. Ligustrazine reportedly is able to relieve pain and suppress inflammation [11-14]. Intra-articular injection of ligustrazine can treat osteoarthritis [12]. And ligustrazine has the protective effect on cartilage and chondrocytes [15]. However, little information is available about the effect of ligustrazine on low back pain and lumbar intervertebral disc degeneration.

In this study, we aimed to examine the effect of ligustrazine on intervertebral disc degeneration. In vivo, a rat model of lumbar spine disc degeneration [16] was used to determine the protective effect of ligustrazine on disc degeneration.

\section{Materials and Methods}

2.1. Drug Preparation. Ligustrazine, another name is tetramethylpyrazine (Figure 1(a)), belongs to pyrazine alkaloids [17]. The ligustrazine hydrochloride in our study was purchased from Nanning Maple Leaf Pharmaceutical Co., Ltd (Nanning, Guangxi province, China (lot number: 051125)). The storage location of the specimen used in our study is the 
<smiles>COc1cc(/C=C/C(=O)O)ccc1O</smiles>

(a)
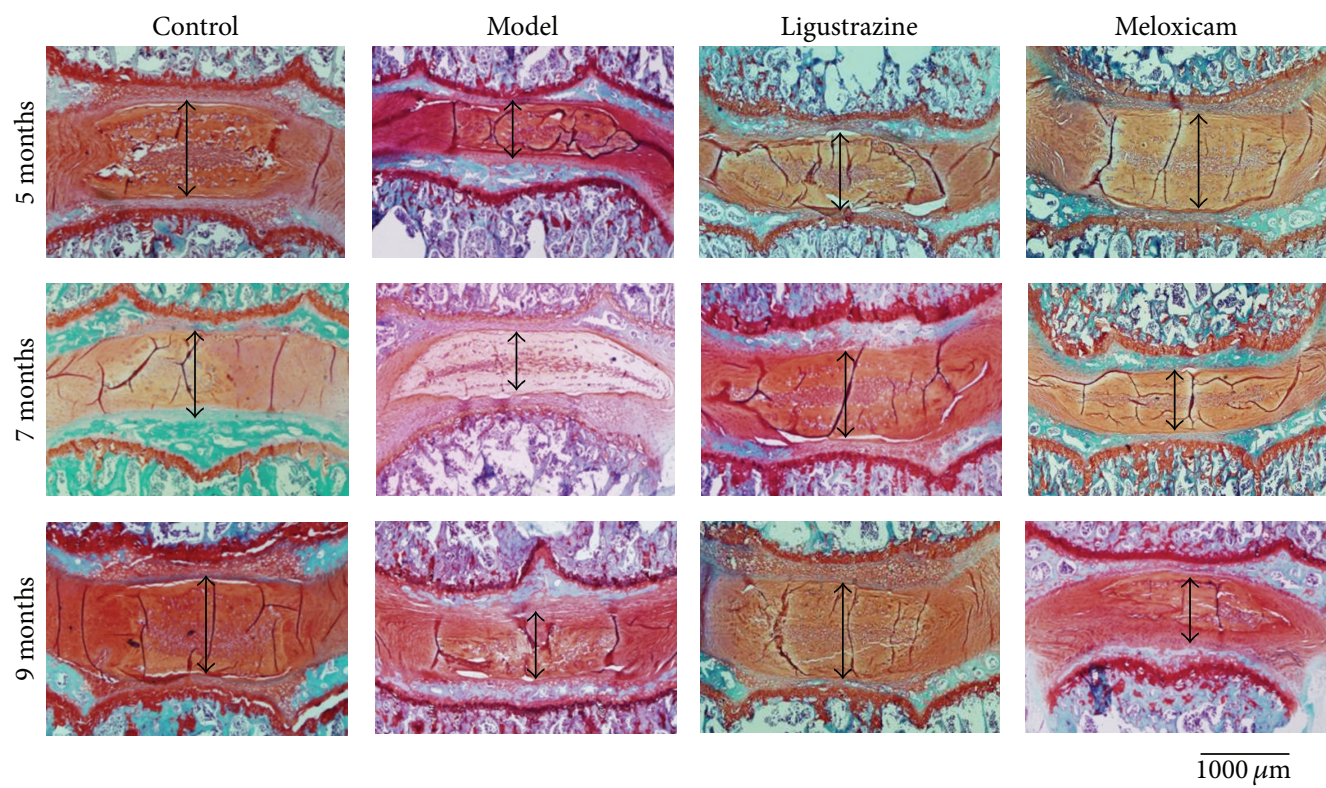

(b)

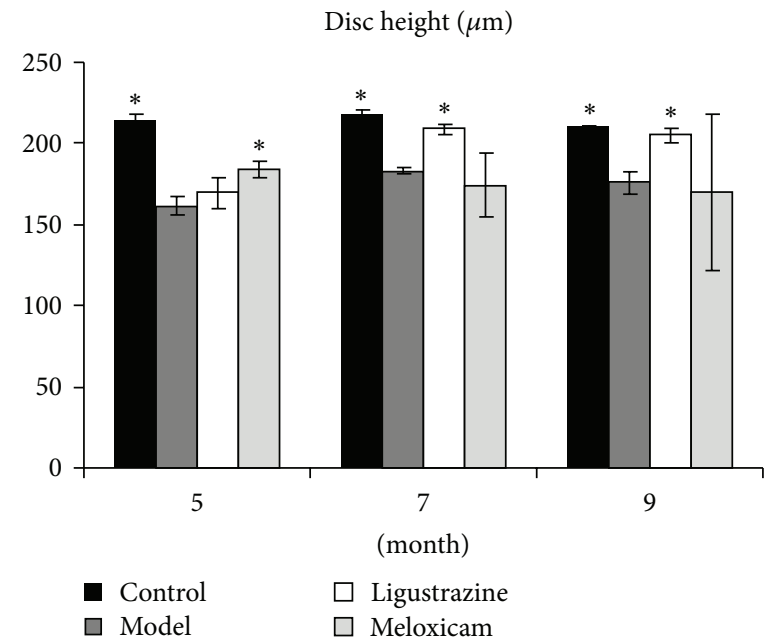

(c)

Figure 1: Chemical structure of ligustrazine and photomicrographs of IVDs stained by safranin-O/fast green. (a) Chemical structure of ligustrazine (tetramethylpyrazine). (b)-(c) Upright posture reduced the heights of IVDs. However, the heights of IVDs increased when ligustrazine pretreatment began seven or nine months after surgery, while meloxicam increased the heights of IVDs when pretreatment started five months after surgery and had no significant effect when pretreatment commenced seven and nine month after surgery. The arrow indicates the quantitative measurements of IVD heights; the bar equals $1000 \mu \mathrm{m}$. Each value represents the mean \pm S.D of 6 sections. ${ }^{*} P<0.05$ compared to model group in the same month. 
TABLE 1: Sequences of primers used in the real-time RT-PCR.

\begin{tabular}{|c|c|c|}
\hline Genes & Forward primer & Reverse primer \\
\hline Col2 $\alpha 1$ (112 bp) & F: $5^{\prime}$-TCCTAAGGGTGCCAATGGTGA-3' & R: 5'-GGACCAACTTTGCCTTGAGGAC-3' \\
\hline MMP 13 (142 bp) & 5'-CCCTGGAGCCCTGATGTTT-3' & 5'-CTCTGGTGTTTTGGGGTGCT-3' \\
\hline Col10 $\alpha 1$ (143 bp) & $5^{\prime}$-TTCACAAAGAGCGGACAGAGA-3' & $5^{\prime}$-TCAAATGGGATGGGAGCA-3' \\
\hline$\beta$-actin (150 bp) & 5'-GGAGATTACTGCCCTGGCTCCTA- ${ }^{\prime}{ }^{\prime}$ & 5'-GACTCATCGTACTCCTGCTTGCTG-3' \\
\hline iNOS (101 bp) & 5'-CTCACTGTGGCTGTGGTCACCTA- ${ }^{\prime}$ & 5'-GGGTCTTCGGGCTTCAGGTTA-3' \\
\hline IL-1 $\beta$ (120 bp) & F: $5^{\prime}$-AGGTCGTCATCATCCCACGAG-3' & R: $5^{\prime}$-GCTGTGGCAGCTACCTATGTCTTG-3' \\
\hline COX-2 (145 bp) & F: $5^{\prime}$-GGAGCATCCTGAGTGGGATGA- $3^{\prime}$ & R: 5'-AAGCAGGTCTGGGTCGAACTTG-3' \\
\hline MMP3 (104 bp) & F: $5^{\prime}$-TGGACCAGGGACCAATGGA-3' & R: 5'-GGCCAAGTTCATGAGCAGCA-3' \\
\hline
\end{tabular}

MMP-13: matrix metalloproteinase 13; IL-1 $\beta$ : interleukin $1 \beta$; COX-2: cyclooxygenase 2; MMP-3: matrix metalloproteinase 3; iNOS: inducible nitric oxide synthase 2 .

institute of Spine, Shanghai University of Traditional Chinese Medicine, China. We used meloxicam, a nonsteroidal antiinflammatory drug (NSAID), as a positive control drug in our experiments because it is widely used as a pain reliever in clinical treatment of intervertebral disc degeneration disease $[18,19]$. We purchased it from Boehringer Ingelheim Shanghai Pharmaceutical Co., Ltd. (national medical license number: H20020217, 7.5 mg/pill).

2.2. Animal Model. All experiments were approved by the Animal Ethics Committee of Shanghai University of Traditional Chinese Medicine. One hundred and twenty onemonth-old male Sprague-Dawley rats (Shanghai Laboratory Animal Center of Chinese Academy of Science) were randomized into the following 12 groups ( $n=10$ for each group): 5-, 7-, and 9-month control group, 5-, 7, and 9-month model group, 5-, 7-, and 9-month ligustrazine group, and 5-, 7-, and 9-month meloxicam group. Rats in the "control" groups underwent no treatment and were raised in ordinary cages.

Both forelimbs of rats in the "model," "ligustrazine," and "meloxicam" groups were amputated from shoulder joints via an anterior approach. And they were forced to keep upright posture in custom-made cages for 5, 7, or 9 months [16]. Four, six, and eight months after the surgery, ligustrazine and meloxicam groups were treated intraperitoneally with ligustrazine hydrochloride injections $(16 \mathrm{~mL} / \mathrm{kg} \cdot \mathrm{d}, 10 \mathrm{~mL}$ sterile saline: $40 \mathrm{mg}$ ligustrazine hydrochloride) or meloxicam $(0.125 \mathrm{mg} / \mathrm{kg} \cdot \mathrm{d})$ for 30 days. The rats from five, seven, and nine month groups were killed at the fifth, seventh, and ninth month after surgery, respectively, and their lumbar spines were harvested for analysis.

2.3. Histological Evaluation. The samples containing the intervertebral discs and adjacent vertebral endplates from L4L5 were fixed (4\% paraformaldehyde; 24 hours), decalcified ( $20 \%$ ethylenediaminetetraacetic acid; $\mathrm{pH} 7.4 ; 21$ days), dehydrated (gradient ethanol), cleared (dimethylbenzene), and then embedded in olefin. At least 4 consecutive $7 \mu \mathrm{m}$ sections were obtained from the sagittal planes and stained (safranin$\mathrm{O} /$ fast green). Sections were examined (photomicroscope set: Olympus DP71; Olympus, Tokyo), and morphometric study was performed (image autoanalysis system: CMIAS99B; Okolab, Milan, Italy).
2.4. Immunostaining for Type II Collagen and IL-1 $\beta$. Tissue sections were dewaxed, cleared, and rehydrated. After incubating in $3 \% \mathrm{H}_{2} \mathrm{O}_{2}$ to block endogenous peroxidase activity for 15 minutes and digestion with protease $\mathrm{K}$ for 10 minutes, the sections were incubated with $5 \%$ bovine serum albumin (BSA) solution in PBS for 1 hour. The sections were then incubated with rabbit polyclonal antibody to type II collagen (Abcam; Cambridge, UK; $1: 100$ dilution) and IL-1 $\beta$ (Abcam; $1: 100$ dilution) at $4^{\circ} \mathrm{C}$ for 12 hours. After thorough wash, the sections were incubated with biotinylated goat anti-rabbit $\operatorname{IgG}$ at $4^{\circ} \mathrm{C}$ for 60 minutes and then with Streptavidin-HRP at $37^{\circ} \mathrm{C}$ for 10 minutes. Color reaction were elicited by $3,3^{\prime}$ diaminobenzidine $(\mathrm{DAB})$ solution. The sections were counterstained with hematoxylin and mounted. Sections were examined using a photomicroscope set (Olympus DP71).

2.5. Total RNA Isolation from Tissue. In accordance with the manufacturer's protocol (Sigma, St. Louis, MO), TRIzol reagent $(1 \mathrm{~mL})$ was used to isolate total RNA from intervertebral disc samples. The isolated RNA was stored at $-80^{\circ} \mathrm{C}$.

2.6. Real-Time PCR Analysis. In accordance with the manufacturer's protocol (advantage RT-for-PCR kit; Takara; Biotechnology Co., Ltd.; Dalian, Liaoning, China), total RNA $(1 \mu \mathrm{g})$ was reverse-transcribed to synthesize cDNA. Quantitative real-time PCR amplifications were carried out in RotorGene real-time DNA amplification system (Corbett Research; Sydney, Australia) using $1 \mu \mathrm{L}$ of cDNA and SYBR Green (BioBad; Hercules, CA) in accordance with the manufacturer's protocol. The primers for Col $2 \alpha 1$, Col10 $\alpha 1$, MMP3, MMP13, IL-1 $\beta$, COX2, iNOS, and $\beta$-actin were designed by TaKaRa Biotechnology Co. Ltd. (see Table 1). Gene expression was normalized to $\beta$-actin and expressed as fold change relative to the expression values in the control groups. PCR products were subjected to melting curve analysis, and the data were quantified using Rotor-Gene 6.0 analysis software.

2.7. Statistical Analysis. Data are expressed as means \pm standard deviation. Statistical analysis was performed (SPSS 10.0; Chicago, IL). One-way ANOVA test was used, followed by Dunnett's test for multiple comparisons. A $P$ value of $<0.05$ was taken as statistically significant. 

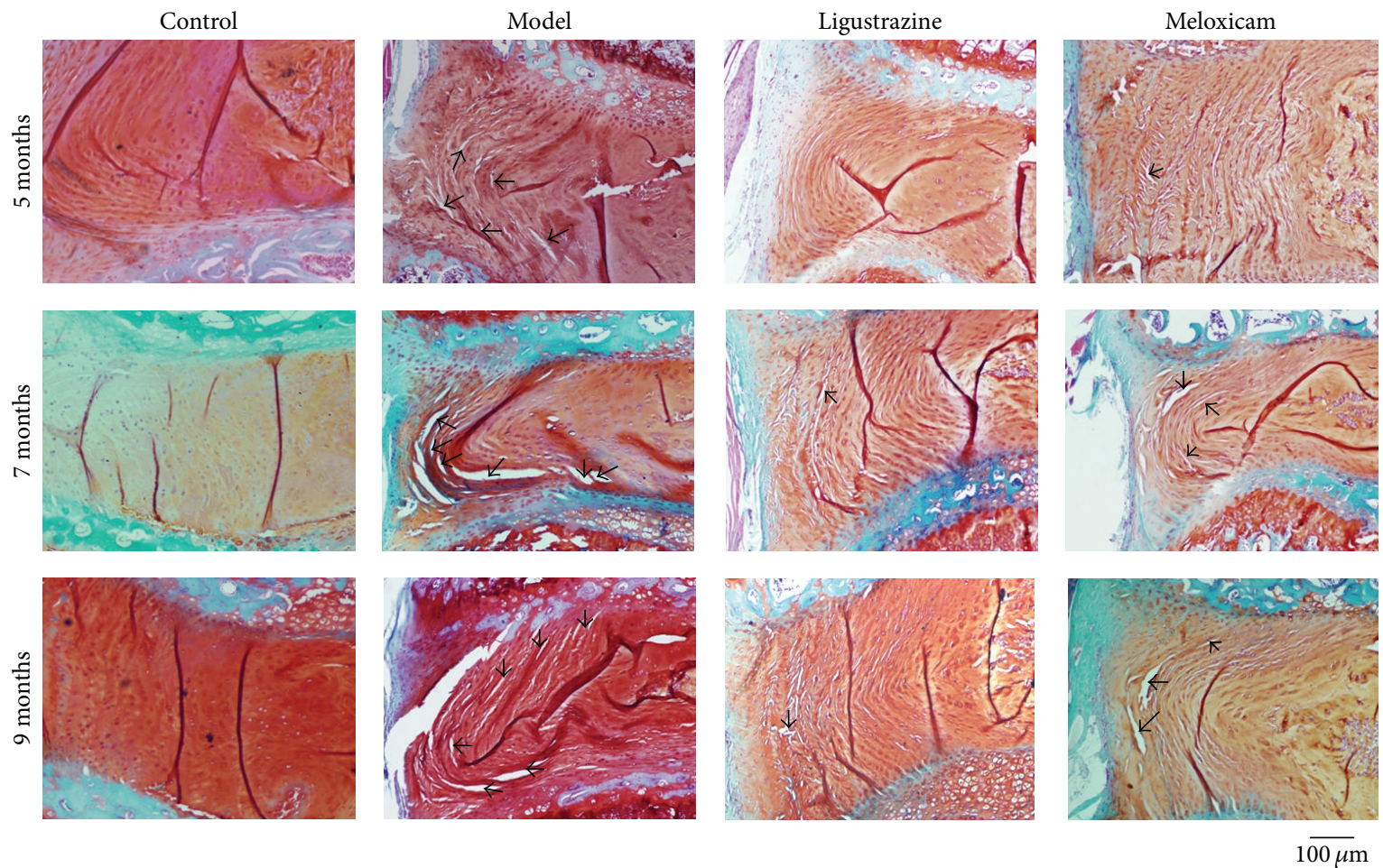

FIgURE 2: Photomicrographs of IVDs (annulus fibrosus) stained by safranin-O/fast green staining. In the surgery group the laminar structures of the annulus fibrosus were fissures at all time points. Ligustrazine almost completely reversed this disorganization at all time points, which has better effect than meloxicam. The arrow indicates the fissures of annulus fibrosus. Bar equals $100 \mu \mathrm{m}$.

\section{Results}

3.1. Histological Observation of the Effect of Ligustrazine on IVD Degeneration. To investigate the effect of ligustrazine on IVD degeneration, we applied an upright posture induced IVD degeneration rat model [16]. We found that in control groups, the intervertebral disc between L4-L5 and adjacent endplates appeared well-organized in annulus fibrosus, endplate, and nucleus pulposus (see Figures 1 and 2). Prolonged upright posture decreased the disc height at all time points. Meloxicam pretreatment could increase the disc height at the fifth month after surgery; it was unsuccessful when pretreatment was postponed to the seventh and ninth month after surgery. The pretreatment with ligustrazine for one month could increase the disc height of upright rats when started seven or nine months after surgery but had no effect when started five months after surgery (see Figure 1).

Prolonged upright posture led to disorganization and fracture of the lamellar architecture of both inner and outer parts of the annulus fibrosus at all time points. When started five or seven months after surgery, ligustrazine pretreatment almost completely reversed the fissures of the inner part of annulus fibrosus subjected to upright posture. When started nine months after surgery, it lessened fissures at all layers of annulus fibrosus-better than the effect of meloxicam (see Figure 2). These results suggested that ligustrazine is an effective treatment on lumbar disc degeneration induced by upright posture.
3.2. Immunohistochemical Findings of the Effect of Ligustrazine on Type II Collagen Protein Expression at Nucleus Pulposus. It was reported that type II collagen is an important component of extracellular matrix of IVDs and plays an essential biomechanical function in the normal disc [18-22]. Thus, we examined type II collagen protein expression by immunohistochemical staining. We found strong immunoreactivity for type II collagen in the nucleus pulposus in control samples. Much weaker immunostaining for type II collagen was observed at any time points in the model group. The density of the type II collagen-positive staining of the ligustrazine group increased dramatically compared to the model group. Meloxicam had no effect on the protein expression of type II collagen (see Figure 3).

\subsection{Ligustrazine Inhibited the Upright Posture-Induced Down-} regulation of Col2 $\alpha 1$ and Upregulation of Col10 $\alpha 1, M M P 13$, and MMP3 mRNA Expression. The protein expression level of type II collagen was affected by its synthesis and catabolism. Therefore, we tested the mRNA expression of Col $2 \alpha 1$ and its degradation enzymes MMP13 and MMP3. We found that the mRNA expression of Col $2 \alpha 1$ was significantly reduced by upright posture at all three time points and that meloxicam pretreatment had no effect on Col $2 \alpha 1$ mRNA expression after surgery. In contrast, the decrease of Col $2 \alpha 1$ mRNA expression was completely recovered by pretreatment with ligustrazine at all time points (see Figure 4(a)). It 

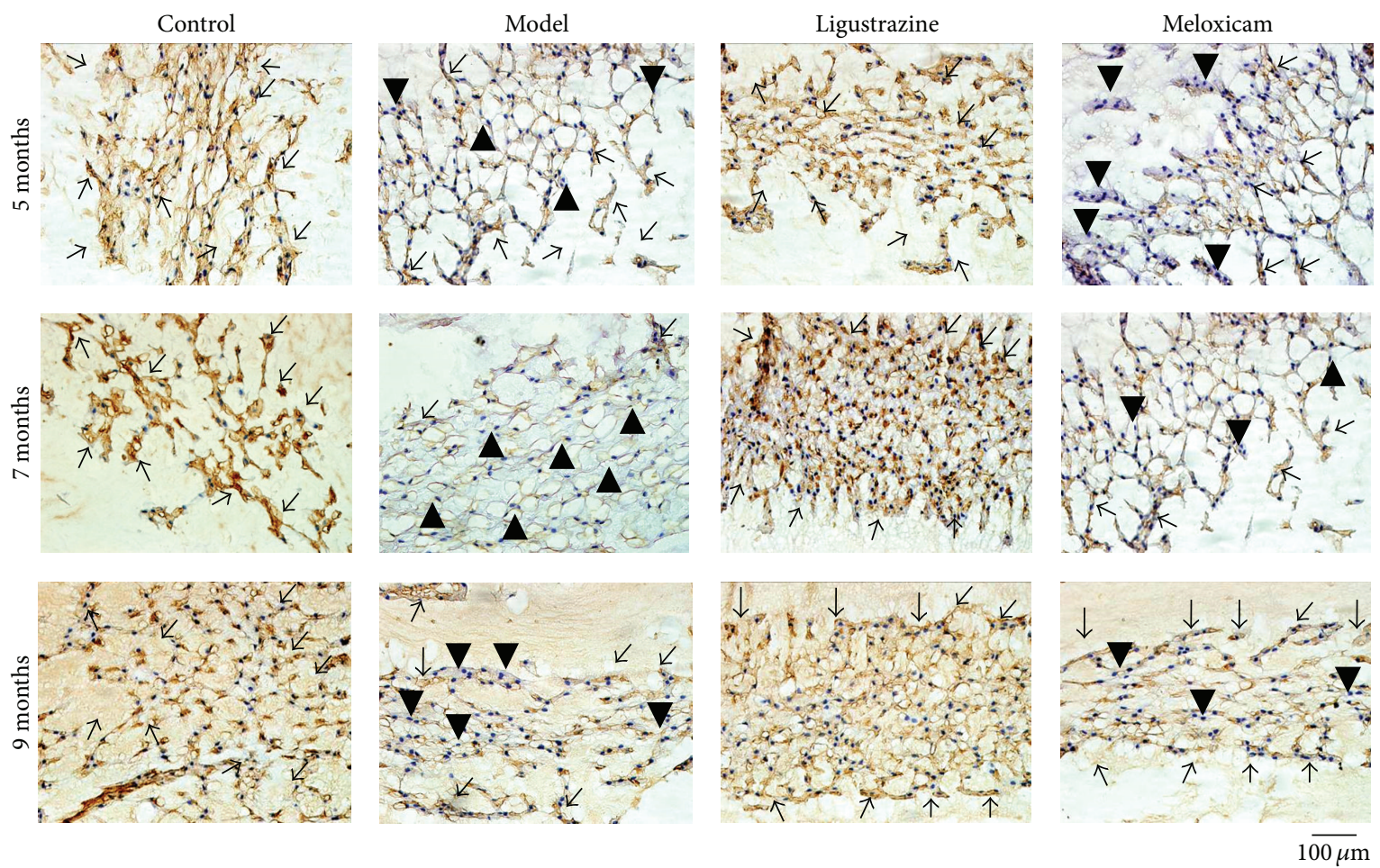

Figure 3: Immunohistochemical assessments of the protein level of type II collagen. Positive staining of type II collagen declined in the nucleus pulposus at all time points after the surgery. Ligustrazine treatment completely reversed the decreased trend of type II collagen at the nucleus pulposus, while meloxicam has no obvious increasing effect. The arrow indicates the positive staining cells; the arrow head indicates the negative staining cells. Bar equals $100 \mu \mathrm{m}$.

indicates that ligustrazine affects type II collagen synthesis directly, while meloxicam has no effect on its synthesis.

On the other hand, upright posture increased mRNA expression of MMP13 and MMP3 at all time points, but both meloxicam and ligustrazine could significantly reverse the upregulation of MMP13 and MMP3 mRNA expression induced by upright posture (see Figures 4(b) and 4(c)). These data suggested that meloxicam and ligustrazine had similar effects when it comes to reducing the degradation of enzyme expression.

It is known that type $\mathrm{X}$ collagen plays an important role in endochondral ossification and matrix calcification of endplate cartilage, and that its increase indicates the terminal stage of intervertebral disc degeneration [23-27]. Thus, we investigated the mRNA expression of Col10 $\alpha 1$, and we found that upright posture increased the mRNA expression of Collo $\alpha 1$ at all time points and meloxicam had no effect on Col10 $\alpha 1$ expression. However, ligustrazine could significantly reverse the upregulation of Col10 $\alpha 1$ mRNA expression induced by upright posture at all time points (Figure 4(d)).

\subsection{Effect of Ligustrazine on IL-1 $\beta$ Protein Distribution. It was} reported that inflammatory cytokines involve the imbalance of synthesis and catabolism of extracellular matrix [28, 29]. Our previous data showed that ligustrazine could improve type II collagen synthesis and inhibit its degradation. We asked ourselves whether ligustrazine has anti-inflammatory effects. We examined the effect of ligustrazine on IL- $1 \beta$ expression and found that in the control groups IL- $1 \beta$ positive staining was very weak and limited at the outer layer of the endplate at all time points. However, in the model groups, there were more IL-1 $\beta$ positive staining cells in the outmost layer of the endplate five and seven months after surgery and distributed to both outer and inner layer of endplate nine months after surgery. Interestingly, immunostaining for this inflammatory mediator was very weak in the ligustrazine groups at both layers of endplate at all time points (see Figure 5).

\subsection{Ligustrazine Blocked Upright Posture Induced Upregula-} tion of IL-1 $\beta$, iNOS, and COX2 mRNA Expression. Finally, we tested the effect of ligustrazine and meloxicam on several inflammation related factors of intervertebral discs. We tested the mRNA expression of IL- $1 \beta$, iNOS, and COX 2 of four 7-month groups and found that long term upright posture increased IL- $1 \beta$, iNOS, and COX 2 mRNA expression. However, this kind of effect was reversed by both ligustrazine and meloxicam pretreatment (Figure 6). Those data revealed that ligustrazine has similar anti-inflammatory effects as meloxicam. 

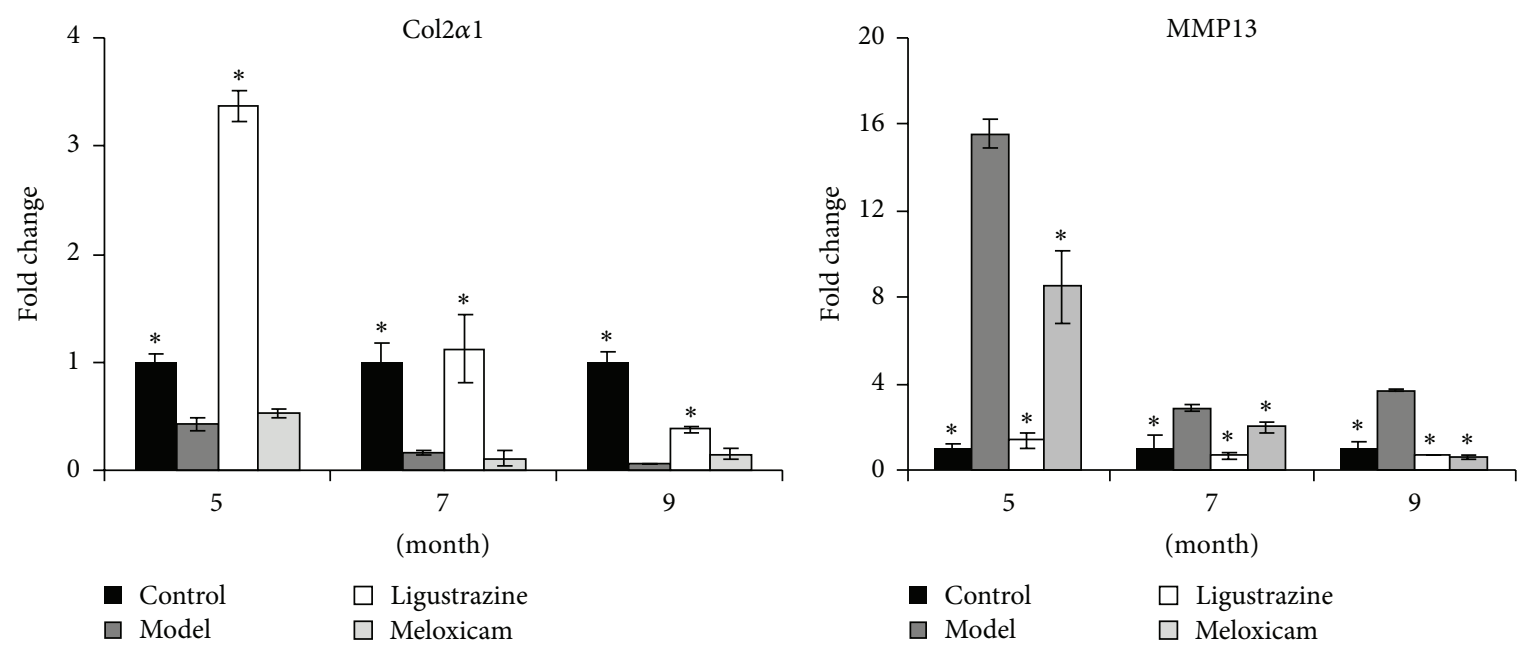

(a)

(b)

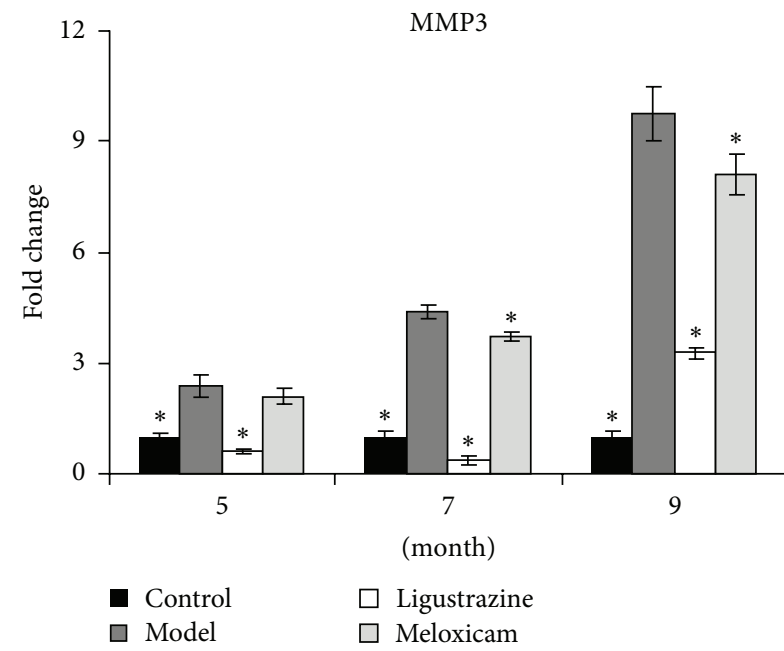

(c)

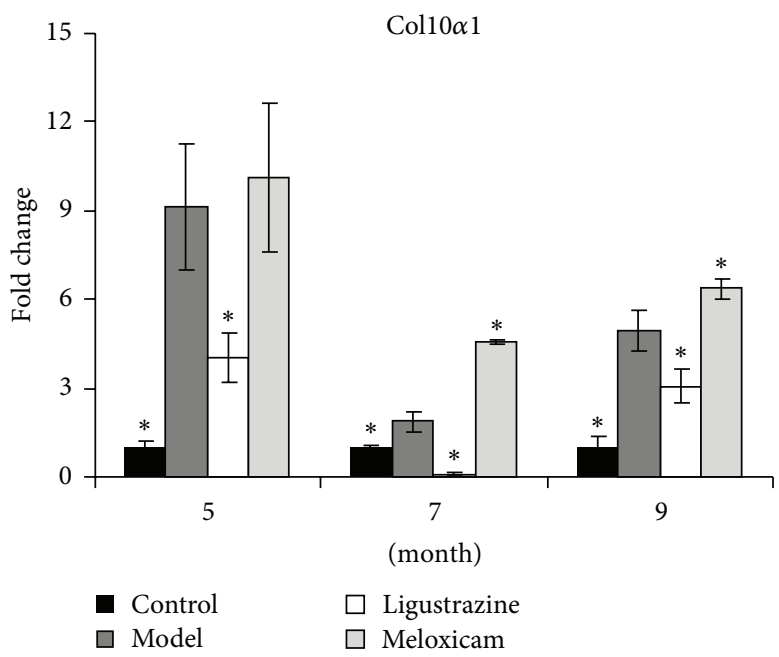

(d)

Figure 4: The mRNA expression of Col2 $\alpha 1$, Col10 $\alpha 1$, MMP3, and MMP13. (a) In the operated rats, the mRNA expression of Col2 $\alpha 1$ decreases significantly at all time points but upregulated significantly after pretreatment with ligustrazine, while meloxicam has no effect on Col $2 \alpha 1$ expression. (b)-(c) MMP3 and MMP13 mRNA expression increased significantly at all time points in model groups, but ligustrazine and meloxicam treatment decreased gene expression. (d) Col10 $\alpha 1 \mathrm{mRNA}$ expression increased significantly at all time points in model groups and ligustrazine treatment decreased gene expression significantly at all time points, while meloxicam has no deducing role on its expression. The columns represent the mean $\pm \mathrm{SD}$ of three independent experiments. ${ }^{*} \mathrm{P}<0.05$ compared to model group in the same month.

\section{Discussion}

In this study, we aimed to determine whether ligustrazine could prevent intervertebral disc degeneration. We therefore carried out an in vivo study to illustrate the effect of ligustrazine in lumbar intervertebral disc degeneration induced by upright posture. We found that ligustrazine could increase the disc height and improve the morphology of the IVDs back to normal, upregulated type II collagen expression, and downregulated degradation enzymes (type $\mathrm{X}$ collagen and inflammatory cytokines expression). These results suggested that ligustrazine is an effective substance against lumbar disc degeneration induced by upright posture.

In our study, we applied meloxicam as positive control, because it was reported that ligustrazine reduces inflammatory response following permanent focal cerebral ischemia [30] and traumatic spinal cord [31], regulates inflammation mediators of cardiovascular disease [32], and inhibits TNF $\alpha$, IL- $1 \beta$, iNOS, NF- $\kappa$ B p65, COX-2, and IL8 expression [33,34]. We sought to compare the different therapeutic effects between ligustrazine and meloxicam. Our study indicates that both meloxicam and ligustrazine could prevent inflammatory mediators' expression in intervertebral discs that meloxicam is better than ligustrazine and that ligustrazine has an anti-inflammation mechanism different from meloxicam. Previous reports revealed that ligustrazine could decrease IL-8 expression by blocking ERK1/2 and p38 phosphorylation [35] and reduce TNF- $\alpha$, iNOS, NF- $\kappa$ B p65, and COX-2 expression by increasing PPAR- $\gamma$ signaling [34]. It was also reported that IL-1-induced ERK phosphorylation 

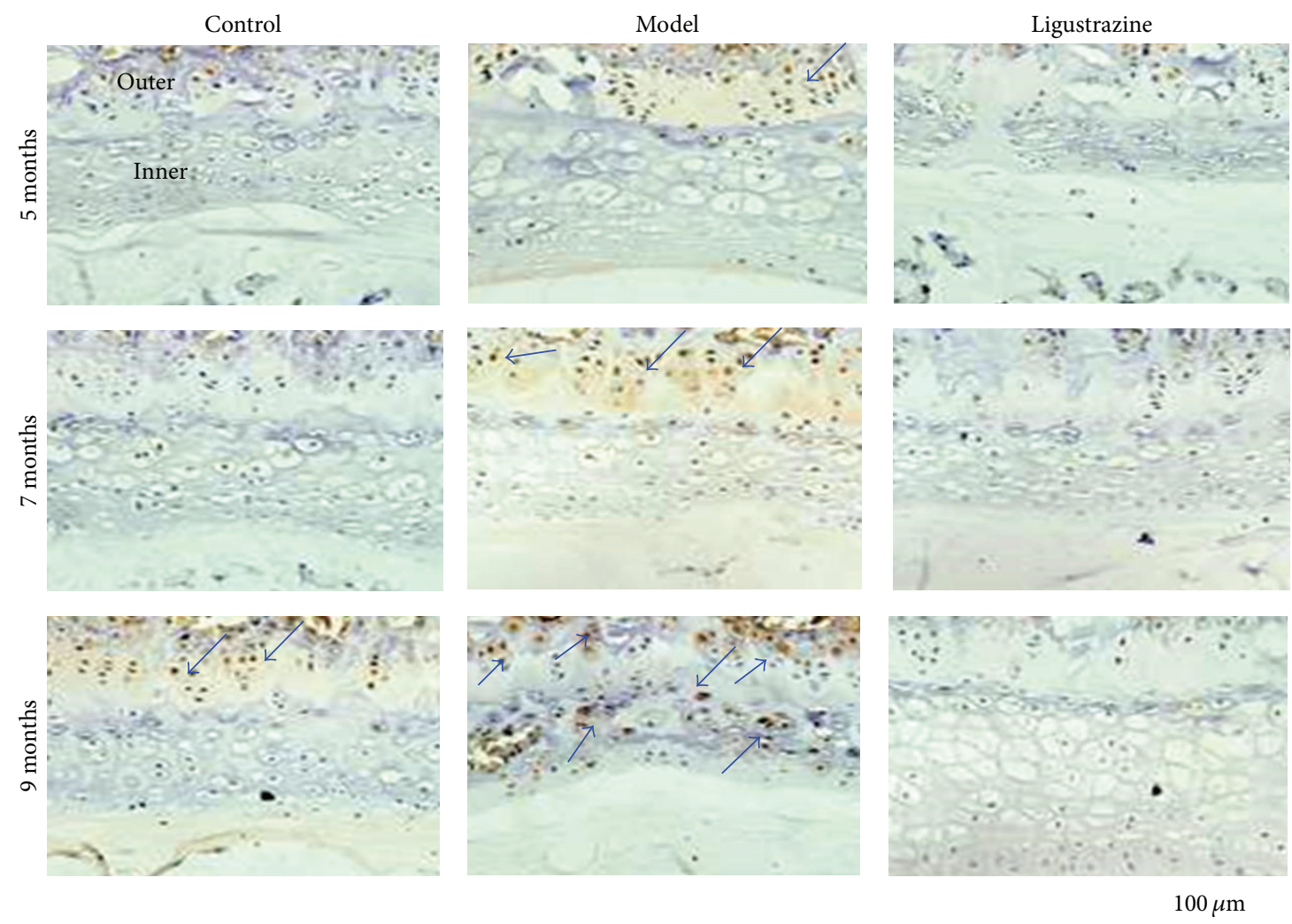

FIGURE 5: Immunohistochemical assessments of the protein level of IL-1 $\beta$. Positive staining of IL- $1 \beta$ increased in the cartilage endplate at all time points after the surgery. Ligustrazine treatment completely blocked the increase of IL-1 $\beta$ at the endplate. Arrows indicate the positive staining cells; outer, the outer layer of endplate; inner, the inner layer of endplate. Bar equals $100 \mu \mathrm{m}$.

is dependent on intracellular $\mathrm{Ca}^{2+}$ signal [36]. Ligustrazine is a calcium antagonist $[18,37,38]$ which could effectively reduce the concentration of calcium. Its calcium antagonist feature might explain ligustrazine's anti-inflammatory effect on chondrocytes.

In addition, meloxicam could only affect the expression of the IL- $1 \beta$ signaling target gene MMP13 and MMP3 but not Col $2 \alpha 1$ and Col10 $\alpha 1$. Moreover, it did not show better effect on intervertebral disc structure while ligustrazine not only reduced degradation enzymes expression but also increased Col $2 \alpha 1$ and reduced Col10 $\alpha 1$ expression. These results suggested that the anti-inflammation effect alone is not enough to counter IVD degeneration diseases; rather, ligustrazine has anti-inflammatory and extracellular matrix synthesis improving effects.

Pretreatment with ligustrazine inhibited Col10 $\alpha 1$ mRNA expression at all time points. This effect suggested that ligustrazine prevents endochondral ossification and matrix calcification at endplate cartilage. It was reported that types $\mathrm{X}$ collagen could interact with cell surface-expressed annexin $\mathrm{V}$; stimulated annexin V-mediated $\mathrm{Ca}^{2+}$ influx, leading to an increased intracellular $\mathrm{Ca}^{2+}$ concentration; and eventually increased alkaline phosphatase activity and mineralization of growth plate chondrocytes [29]. Exogenous extracellular calcium could induce the synthesis of type $\mathrm{X}$ collagen in chondrocytes [39]. Ligustrazine, a calcium antagonist, blocks not only the entry of extracellular calcium through calcium channels but also the release of intracellular stored calcium $[18,37,38]$. This suggested that the inhibitory effect of ligustrazine on type $\mathrm{X}$ collagen expression might be due to its calcium channel blocker characteristic.

Besides that, ligustrazine could regulate capillary permeability and microcirculatory perfusion [40, 41], has antioxidation effect $[41,42]$ and the ability to penetrate the blood-brain barrier [43]. Whether all those abilities of ligustrazine are involved in its protective effect on IVD degeneration deserves further research.

For the first time, ligustrazine was demonstrated to be effective in the lumbar intervertebral disc degeneration induced by prolonged upright posture. The findings in this study suggest that ligustrazine is a promising drug candidate for the therapy of IVD degeneration related diseases.

\section{Conflict of Interests}

The authors declare that there is no conflict of interests regarding the publication of this paper.

\section{Acknowledgments}

National Basic Research Program of China (973 Program 2010CB530400), Ministry of Education, "Innovative 


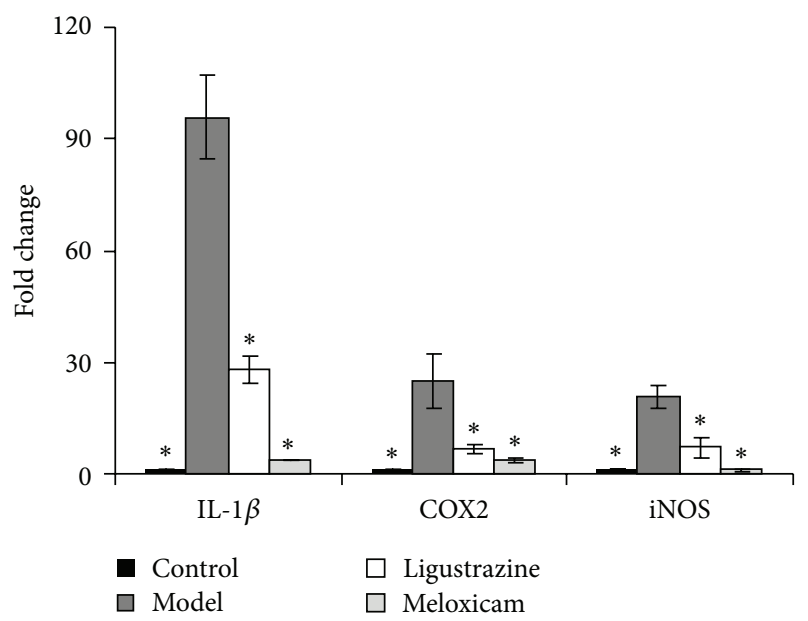

FIgURE 6: The mRNA expression of IL-1 $\beta$, iNOS, and COX 2 of intervertebral discs from the 7-month groups. Compared with control groups, upright posture increased mRNA expression of IL$1 \beta$, iNOS, and COX2 significantly, but ligustrazine and meloxicam pretreatment downregulated those gene expression. The columns represent the mean $\pm \mathrm{SD}$ of three independent experiments. ${ }^{*} P<$ 0.05 compared to model group.

Research Team" (IRT1270), National Natural Science Foundation of China (81102606, 81330085, 81220108027, and 81173278), Higher National Excellent Doctoral Dissertation of projects funded projects (201276), Natural Science Foundation of Shanghai (11ZR1437100), Shanghai Youth Science and Technology Star Program (12QA1403200), National Clinical Research Base "Long Medical Scientists, Long Medical Team" Project (LYTD-01), Shanghai University of Traditional Chinese Medicine "085 Project" (085ZY1204) are gratefully acknowledged. Our experimental work was reviewed by Institutional Animal Ethics Committee of Shanghai University of Traditional Chinese Medicine.

\section{References}

[1] B. G. Peng, "Pathophysiology, diagnosis, and treatment of discogenic low back pain," World Journal of Orthopaedics, vol. 4, no. 2, pp. 42-52, 2013.

[2] F. Ding, Z. W. Shao, and L. M. Xiong, "Cell death in intervertebral disc degeneration," Apoptosis, vol. 18, no. 7, pp. 777-785, 2013.

[3] B. Morlion, "Chronic low back pain: pharmacological, interventional and surgical strategies," Nature Reviews Neurology, vol. 9, no. 8, pp. 462-473, 2013.

[4] T. Pincus, S. Anwar, L. McCracken et al., "Testing the credibility, feasibility and acceptability of an optimised behavioural intervention (OBI) for avoidant chronic low back pain patients: protocol for a randomised feasibility study," Trials, vol. 14, article $172,2013$.

[5] S. Ren, Y. Liu, J. Ma et al., "Treatment of rabbit intervertebral disc degeneration with co-transfection by adeno-associated virus-mediated SOX9 and osteogenic protein-1 double genes in vivo," International Journal of Molecular Medicine, vol. 32, no. 5, pp. 1063-1068, 2013.
[6] P. Colombier, J. Clouet, O. Hamel, L. Lescaudron, and J. Guicheux, "The lumbar intervertebral disc: from embryonic development to degeneration," Joint Bone Spine, vol. 81, no. 2, pp. 125-129, 2014.

[7] H. G. Xu, J. F. Cheng, H. X. Peng et al., "JNK phosphorylation promotes natural degeneration of cervical endplate chondrocytes by down-regulating expression of ANK," European Review for Medical and Pharmacological Sciences, vol. 17, no. 17, pp. 2335-2344, 2013.

[8] Y. Wu, S. Cisewski, B. L. Sachs, and H. Yao, "Effect of cartilage endplate on cell based disc regeneration: a finite element analysis," Molecular and Cellular Biomechanics, vol. 10, no. 2, pp. 159-182, 2013.

[9] M. M. Katz, A. R. Hargens, and S. R. Garfin, "Intervertebral disc nutrition. Diffusion versus convection," Clinical Orthopaedics and Related Research, vol. 210, pp. 243-245, 1986.

[10] J. P. G. Urban, S. Smith, and J. C. T. Fairbank, "Nutrition of the intervertebral disc," Spine, vol. 29, no. 23, pp. 2700-2709, 2004.

[11] X. W. Che, Y. Zhang, H. Wang, and W. Wang, "Effect of ligustrazine injection on levels of interleukin- 4 and interferon- $\gamma$ in patients with bronchial asthma," Chinese Journal of Integrative Medicine, vol. 14, no. 3, pp. 217-220, 2008.

[12] J. Z. Hu, C. Y. Luo, M. Kang, H. B. Lü, G. H. Lei, and Z. Dai, "Therapeutic effects of intraarticular injection of ligustrazine on knee osteoarthritis," Zhong Nan Da Xue Xue Bao Yi Xue Ban, vol. 31, no. 4, pp. 591-594, 2006.

[13] X. P. Zhang, D. R. Liu, and Y. Shi, "Study progress in therapeutic effects of traditional Chinese medicine monomer in severe acute pancreatitis," Journal of Zhejiang University Science B, vol. 8, no. 2, pp. 147-152, 2007.

[14] Y. F. Leng, X. M. Gao, S. X. Wang, and Y. H. Xing, "Effects of tetramethylpyrazine on neuronal apoptosis in the superficial dorsal horn in a rat model of neuropathic pain," The American Journal of Chinese Medicine, vol. 40, no. 6, pp. 1229-1239, 2012.

[15] X. D. Ju, M. Deng, Y. F. Ao et al., "The protective effect of tetramethylpyrazine on cartilage explants and chondrocytes," Journal of Ethnopharmacology, vol. 132, no. 2, pp. 414-420, 2010.

[16] Q. Q. Liang, Q. Zhou, M. Zhang et al., "Prolonged upright posture induces degenerative changes in intervertebral discs in rat lumbar spine," Spine, vol. 33, no. 19, pp. 2052-2058, 2008.

[17] H. B. Li and F. Chen, "Preparative isolation and purification of chuanxiongzine from the medicinal plant Ligusticum chuanxiong by high-speed counter-current chromatography," Journal of Chromatography A, vol. 1047, no. 2, pp. 249-253, 2004.

[18] P. K. T. Pang, J. J. Shan, and K. W. Chiu, "Tetramethylpyrazine, a calcium antagonist," Planta Medica, vol. 62, no. 5, pp. 431-435, 1996.

[19] T. R. Oegema Jr., "The role of disc cell heterogeneity in determining disc biochemistry: a speculation," Biochemical Society Transactions, vol. 30, part 6, pp. 839-844, 2002.

[20] P. J. Roughley, "Biology of intervertebral disc aging and degeneration: involvement of the extracellular matrix," Spine, vol. 29, no. 23, pp. 2691-2699, 2004.

[21] J. I. Sive, P. Baird, M. Jeziorsk, A. Watkins, J. A. Hoyland, and A. J. Freemont, "Expression of chondrocyte markers by cells of normal and degenerate intervertebral discs," Journal of Clinical Pathology-Molecular Pathology, vol. 55, no. 2, pp. 91-97, 2002.

[22] C. L. Yang, H. Rui, S. Mosler, H. Notbohm, A. Sawaryn, and P. K. Muller, "Collagen II from articular cartilage and annulus fibrosus. Structural and functional implication of tissue specific posttranslational modifications of collagen molecules," 
European Journal of Biochemistry, vol. 213, no. 3, pp. 1297-1302, 1993.

[23] T. Aigner, K. Greskötter, J. C. T. Fairbank, K. von der Mark, and J. P. G. Urban, "Variation with age in the pattern of type $\mathrm{X}$ collagen expression in normal and scoliotic human intervertebral discs," Calcified Tissue International, vol. 63, no. 3, pp. 263-268, 1998.

[24] N. Boos, A. G. Nerlich, I. Wiest, K. von der Mark, and M. Aebi, "Immunolocalization of type X collagen in human lumbar intervertebral discs during ageing and degeneration," Histochemistry and Cell Biology, vol. 108, no. 6, pp. 471-480, 1997.

[25] M. E. Grant, "From collagen chemistry towards cell therapy-a personal journey," International Journal of Experimental Pathology, vol. 88, no. 4, pp. 203-214, 2007.

[26] S. Roberts, M. A. Bains, A. Kwan, J. Menage, and S. M. Eisenstein, "Type X collagen in the human invertebral disc: an indication of repair or remodelling?" Histochemical Journal, vol. 30, no. 2, pp. 89-95, 1998.

[27] Y. M. Xi, Y. G. Hu, Z. H. Lu, H. J. Zheng, Y. Chen, and Z. Qi, "Gene expression of collagen types IX and X in the lumbar disc," Chinese Journal of Traumatology, vol. 7, no. 2, pp. 76-80, 2004.

[28] C. P. de Oliveira, L. M. Rodrigues, M. V. Fregni, A. Gotfryd, A. M. Made, and M. A. Pinhal, "Extracellular matrix remodeling in experimental intervertebral disc degeneration," Acta Ortopédica Brasileira, vol. 21, no. 3, pp. 144-149, 2013.

[29] H. Li, C. Liang, Q. Chen, and Z. Yang, "Rhein: a potential biological therapeutic drug for intervertebral disc degeneration," Medical Hypotheses, vol. 77, no. 6, pp. 1105-1107, 2011.

[30] T. K. Kao, C. Y. Chang, Y. C. Ou et al., “Tetramethylpyrazine reduces cellular inflammatory response following permanent focal cerebral ischemia in rats," Experimental Neurology, vol. 247, pp. 188-201, 2013.

[31] J. Z. Hu, J. H. Huang, Z. M. Xiao, J. H. Li, X. M. Li, and $\mathrm{H}$. B. Lu, "Tetramethylpyrazine accelerates the function recovery of traumatic spinal cord in rat model by attenuating inflammation," Journal of the Neurological Sciences, vol. 324, no. 1-2, pp. 94-99, 2013.

[32] L. Guo, A. Wang, Y. Sun, and C. Xu, "Evaluation of antioxidant and immunity function of tetramethylpyrazine phosphate tablets in vivo," Molecules, vol. 17, no. 5, pp. 5412-5421, 2012.

[33] G. G. Yeom, S. Min, and S. Y. Kim, “2,3,5,6-Tetramethylpyrazine of Ephedra sinica regulates melanogenesis and inflammation in a UVA-induced melanoma/keratinocytes co-culture system," International Immunopharmacology, vol. 18, no. 2, pp. 262-269, 2014.

[34] X. He, Z. Zheng, X. Yang, Y. Lu, N. Chen, and W. Chen, "Tetramethylpyrazine attenuates PPAR- $\gamma$ antagonistdeteriorated oxazolone-induced colitis in mice," Molecular Medicine Reports, vol. 5, no. 3, pp. 645-650, 2012.

[35] J. Yin, C. Yu, Z. Yang et al., "Tetramethylpyrazine inhibits migration of SKOV3 human ovarian carcinoma cells and decreases the expression of interleukin- 8 via the ERK1/2, p38 and AP-1 signaling pathways," Oncology Reports, vol. 26, no. 3, pp. 671-679, 2011.

[36] Q. Wang, G. P. Downey, C. Choi, A. Kapus, and C. A. McCulloch, "IL-1 induced release of $\mathrm{Ca}^{2+}$ from internal stores is dependent on cell-matrix interactions and regulates ERK activation," The FASEB Journal, vol. 17, no. 13, pp. 1898-1900, 2003.
[37] C. Y. Kwan, "Plant-derived drugs acting on cellular $\mathrm{Ca}^{2+}$ mobilization in vascular smooth muscle: tetramethylpyrazine and tetrandrine," Stem Cells, vol. 12, no. 1, pp. 64-67, 1994.

[38] Z. Ren, J. Ma, P. Zhang et al., "The effect of ligustrazine on L-type calcium current, calcium transient and contractility in rabbit ventricular myocytes," Journal of Ethnopharmacology, vol. 144, no. 3, pp. 555-561, 2012.

[39] D. K. Bonen and T. M. Schmid, "Elevated extracellular calcium concentrations induce type $\mathrm{X}$ collagen synthesis in chondrocyte cultures," Journal of Cell Biology, vol. 115, no. 4, pp. 1171-1178, 1991.

[40] M. Minamiyama and L. Chang, "Macro- and microcirculatory effects of tetramethylpyrazine and extract of Charthamus tinctorius L. in rabbit mesentery," Clinical Hemorheology and Microcirculation, vol. 23, no. 2-4, pp. 153-158, 2000.

[41] Q. F. Xue, S. L. Dai, S. Y. Yuan et al., "Effect of Chuanxiongqin (tetramethylpyrazine) on microcirculatory perfusion in hamsters and capillary permeability in rats," Proceedings of the Chinese Academy of Medical Sciences and the Peking Union Medical College, vol. 4, no. 4, pp. 224-228, 1989.

[42] H. Wan, H. Zhu, M. Tian et al., "Protective effect of chuanxiongzine-puerarin in a rat model of transient middle cerebral artery occlusion-induced focal cerebral ischemia," Nuclear Medicine Communications, vol. 29, no. 12, pp. 1113-1122, 2008.

[43] T. H. Tsai and C. Liang, "Pharmacokinetics of tetramethylpyrazine in rat blood and brain using microdialysis," International Journal of Pharmaceutics, vol. 216, no. 1-2, pp. 61-66, 2001. 


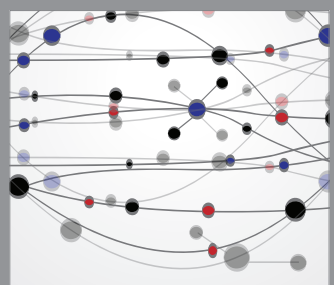

The Scientific World Journal
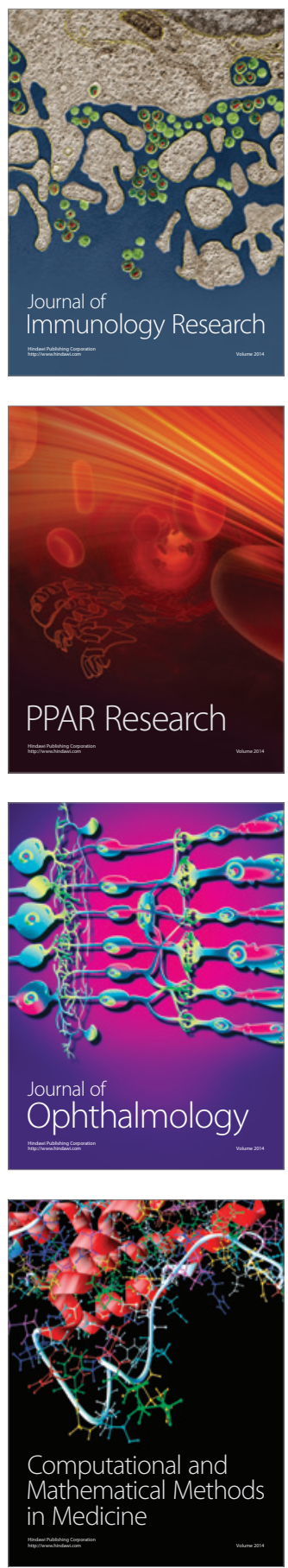

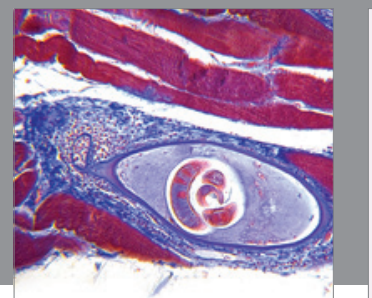

Gastroenterology

Research and Practice
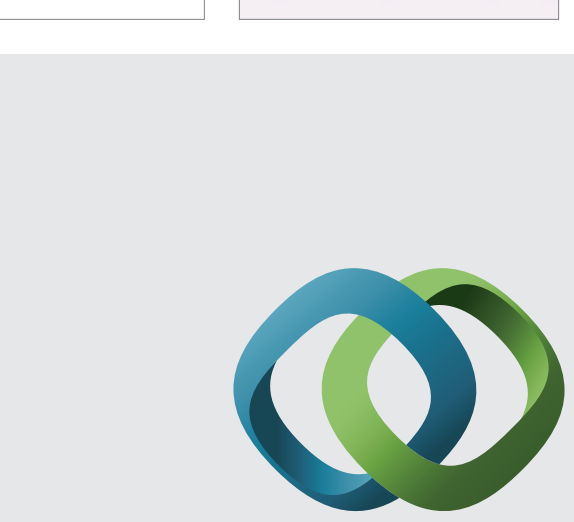

\section{Hindawi}

Submit your manuscripts at

http://www.hindawi.com
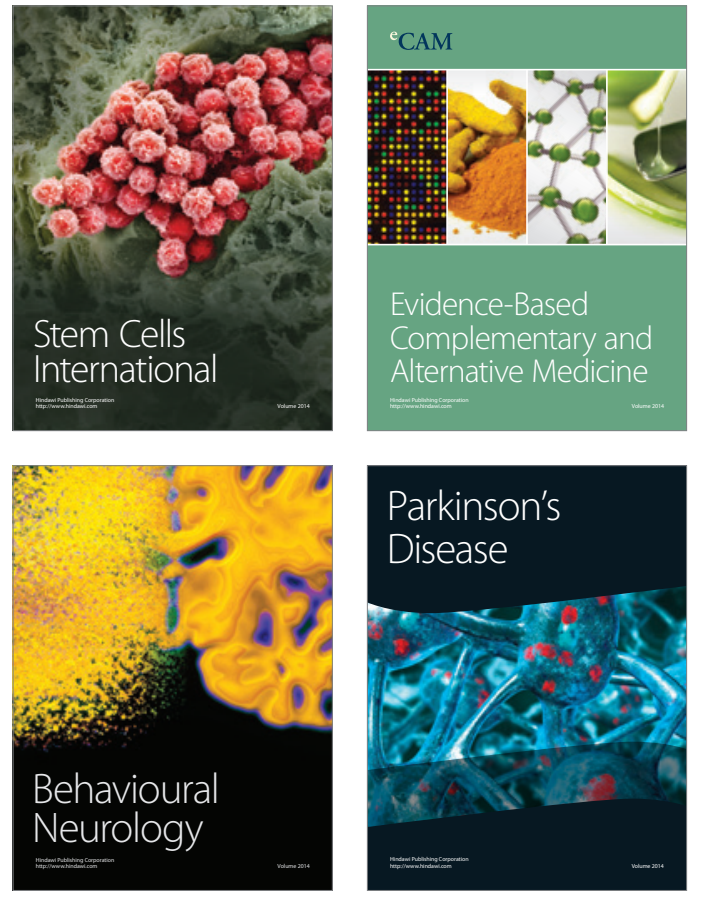
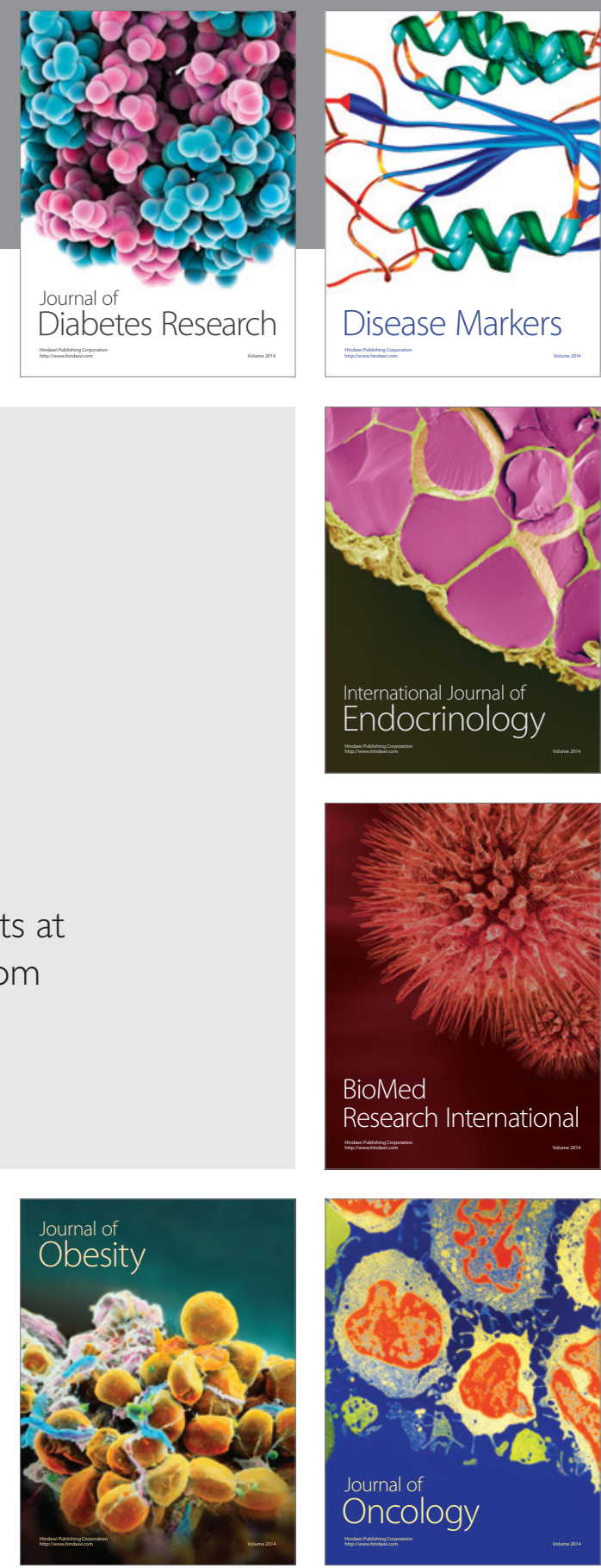

Disease Markers
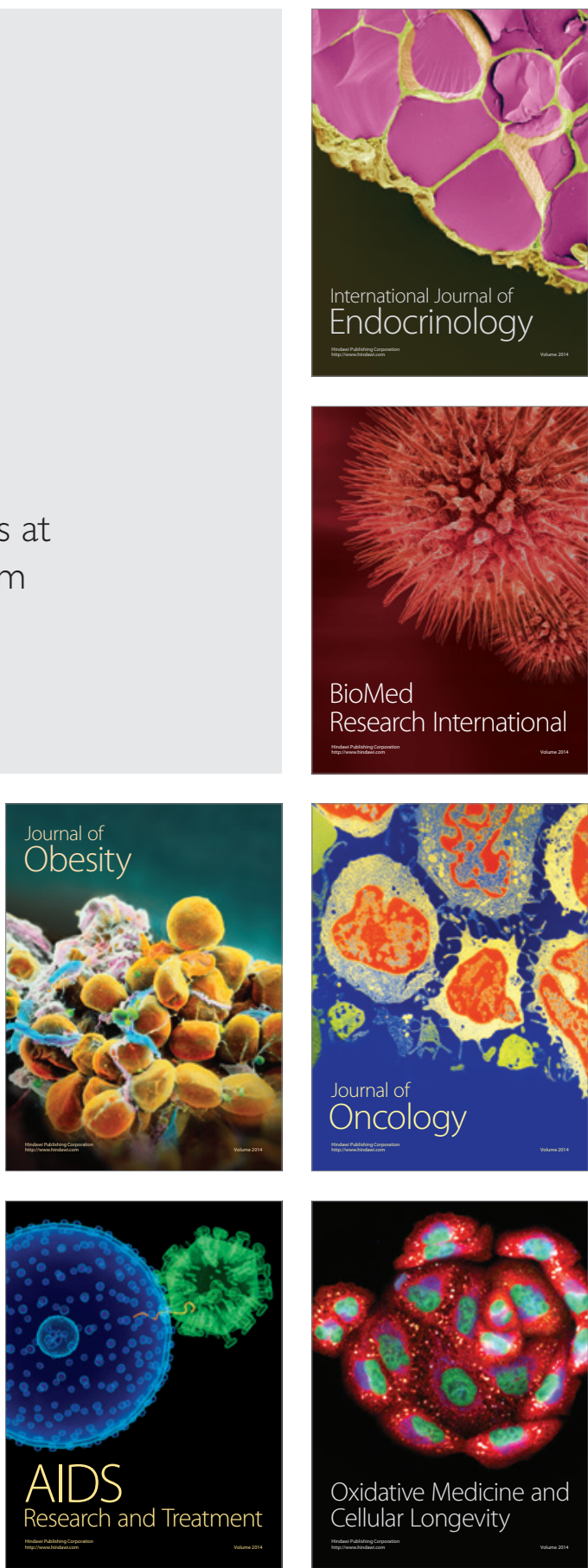